\title{
A MODIFICATION OF THE SMITH INDIAN TECHNIQUE OF INTRACAPSULAR CATARACT OPERATION*
}

\author{
BY \\ M. S. NIRANKARI AND M. C. MAUDGAL \\ Department of Ophthalmology, Medical College, Amritsar, India
}

THE best results of cataract surgery are achieved by the intracapsular technique. McNamara (1868) practised this procedure; he would make the incision in the lower half of the cornea and apply pressure with the finger from above. Molroney, working at Amritsar and Jullundur, extended this procedure to all types of senile cataract. His successor Smith (1928) noticed that the rate of vitreous prolapse was insignificant where the lens was tumbled accidentally; he developed this technique of tumbling as a routine measure, mainly in the soft lens (Morgagnian), and later extended this method to the hard lens. This tumbling is now the essential step in all modern intracapsular surgical procedures.

The Smith Indian method depends upon the application of pressure to the outside of the eyeball to rupture the zonule and to extract the lens by the continued application of pressure; the position and direction of the pressure must be changed as the lens is manoeuvred into its various positions. The hydrostatic principle behind this technique depends upon the equalization of pressure in all directions when the pressure is applied to a fluid contained in a distensible sac. The backward-directed pressure is applied to all the structures behind the diaphragm of the lens, its suspensory ligament, and ciliary processes, and as this diaphragm is the weakest part in the scleral shell it must break. The zonular fibres being the most fragile, the break occurs there first. Application of pressure with the hook controls this break, the ideal site being from below, with the superior portion remaining hinged. Because of this the continuation of pressure tilts out the freed lower equator of the lens, compelling it to go upwards and anteriorly through a rotation of nearly $180^{\circ}$; this tumbling thus causes the lower part of the lens to present first in the corneo-scleral incision. The upper part of the lens, which is still attached to the zonule, acts as an effective cork in the bottle-neck against the prolapse of the vitreous.

Smith achieved this end by applying the knee of the hook to the sclera well down in the inferior fornix, the ball of the hook touching the globe behind the edge of the lens. The broad spatula was held with its convex surface against the posterior lip of the section. Pressure was now exerted with the spatula against the cornea over the section, the hook (both knee

* Received for publication January 7, 1957. 
and ball) being directed towards the centre of the globe. Slowly the hook was advanced towards the sclero-corneal margin, until the edge of the lens was reached and raised. The pressure on the hook was released, and the ball was raised over the conjunctival roll. The spatula was removed and the hook, continuing its course, tucked the cornea under the lens. The lens, still attached along its upper border, was then gently raked off.

\section{Modification of the Smith Indian Technique}

The classical Smith procedure produces vitreous prolapse in a large percentage of cases in inexpert hands. Smith himself was endeavouring to find out a method of lens expression without this shortcoming, and we have continued the efforts in this direction and have developed a technique which is much safer and is easily learned by the individual surgeon. The incidence of vitreous prolapse is not greater than with other procedures. The classical Smith operation has been abandoned by most surgeons, and we have found the modified technique to be very advantageous. It is a speedy procedure which enables us to handle fifty or sixty cases a day during the peak season (February to April and September to November each year). There is a minimum of instrumentation, and only the iris forceps and iris repositor are introduced into the anterior chamber.

Basal narcosis and van Lint's akinesia provide perfect control of the patient and his eyeball. We seldom feel the necessity of eye speculum; it may be dangerous if the fornices are contracted, and will exert pressure on the eyeball which may lead to or predispose to vitreous prolapse.

A limbal incision of $180^{\circ}$ with a narrow von Graefe knife is followed by peripheral button-hole iridectomy (sometimes two or three are done). A good wide incision is the first necessity. The mechanism of this improved and modified technique may be described in three stages: lever, ledge, and tumble.

(1) Lever.-With the convex surface of the lens spatula applied against the upper limbus (Fig. 1) over the corneo-scleral incision, pressure is exerted downwards and backwards on the upper equatorial region of the lens; the vertical diameter of the lens acts as the arm of the lever, the upper part of the lens as the fulcrum. This results in the tilting forward of the lower periphery of the lens. This produces a slight bulge at the lower limbus and indicates the exact position of the lower equator of the lens. The operator now places a hook below this bulge with its point directed posteriorly and tilting upwards (Fig. 2). A gentle pressure with the point breaks the zonule fibres, already stretched by the tilting forward of the lower edge of the lens.

(2) Ledge.-As this happens, the lower border of the lens becomes free, forming a ledge at the limbus. A slight dimple in the sclera below this point indicates its position. The hook is now placed in the gutter below the ledge and the lens is held as it were in a vice, between the lens spatula above and the lens hook below (Fig. 3). 


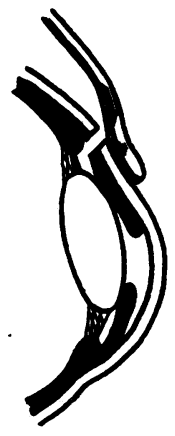

1

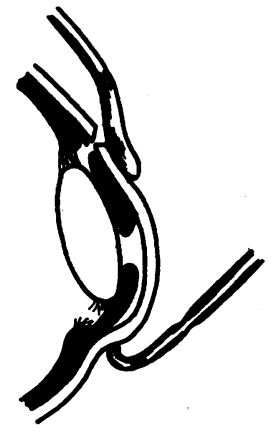

2

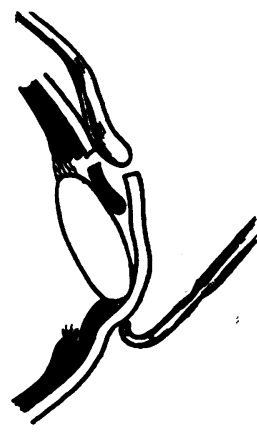

3

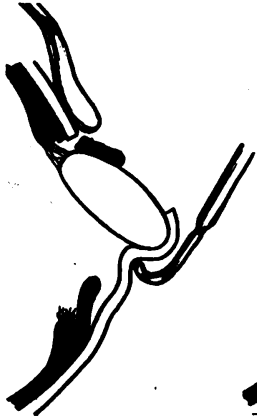

4

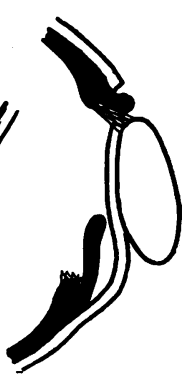

5

(3) Tumble.-Very gentle backward and downward pressure is maintained at the upper edge of the lens while the lens hook gently presses the lower edge of the lens upwards and forwards. The lens begins to tumble, the force being at the back of the equator of the lens and directed upwards and forwards. The fibres of the suspensory ligament in the lower nasal and temporal part are gently stretched and with gentle force they are broken. Now the lens starts rotating forwards around its horizontal diameter, being freed from the attachment of the suspensory ligament (Fig. 4). The spatula keeps the eye steady and also protects the upper zonular fibre from breaking prematurely.

We extract the lens through a moderately dilated pupil. The dilatation is effected by cocaine, adrenaline drops, and retrobulbar anaesthesia; atropine or homatropine are not used. The protection to the vitreous by the iris diaphragm is lost if the pupil is widely dilated.

The whole process of lens delivery can be compared to the delivery of a foetus; instead of a normal vertex presentation we aim at a breech delivery by external version.

It is most interesting to watch the lower equator of the lens engaging the pupil and steadily pressing the pupillary area; in the process the pupil becomes dilated and the lens itself is slightly moulded. In some cases a permanent impression in the form of a slight bulge with a constriction behind forms at the lower edge of the lens and gives it a resemblance to caput succidanum. The slow dilatation of the pupil is just like the opening of the os cervix in a case of obstetric labour. When the largest diameter of the lens is through the pupil, the pressure with the hook may be relaxed a little, the major part of the lens lying in the anterior chamber and part of it being outside engaged in the corneo-scleral wound. Now, with a smooth upward sweep of the hook, the lens is brought out; in some cases where the eye is hypotonic the cornea springs behind the lens, forming a cup or saucershaped depression in which the lens rests, being still attached at its upper edge to the zonular fibres (Fig. 5). The lens is swept aside and the upper zonular fibres are stripped off in this process.

The whole process is based on the simple mechanism of lever action. The surgeon is working all the time on the anterior segment of the eye. No force whatsoever is exerted on the vitreous humour and the posterior segment. The various steps in the delivery of the lens are confined to the anterior segment and its connecting zonular fibres. 
The iris is then reposited with the iris repositor and the corneal flap is reposed to its normal position. The incision line is pressed gently with the cotton wool swab for perfect apposition of the corneo-scleral wound. When this procedure is carried out in a calm and confident manner, the globe and its contents are absolutely under the control of the operator.

This simple and effective procedure for the extraction of the lens seldom fails if the proper indications exist.

The following criteria must be fulfilled before one attempts this technique:

(1) Age.-This procedure can only be adopted in patients above 50 years of age, in whom the zonular ligaments are brittle and fragile. It should never be done in a case under 45 years of age. The friability of the zonular fibres depends on the patient's age and not on the maturity or otherwise of the cataract.

(2) The lens should be situated at its normal place and no part of the zonular ligaments should be already broken. Subluxation of the lens is a contraindication.

(3) The ocular tension should be within the normal limits (18 to $25 \mathrm{~mm}$. $\mathrm{Hg}$ ).

(4) The lens should be fairly hard. In cases of Morgagnian cataract the lever mechanism is not very effective as the pressure with the hook and the spoon cannot be properly transmitted to the zonular fibres at the lower equatorial region. Because of the semifluid nature of the cortex, the force which is desired and directed along the vertical direction is transmitted in expanding the lens in horizontal direction. It thus takes longer to break the suspensory ligament of the lower part, but once the tilting of the lens starts, the rest of the procedure does not differ from that described above. The capsule containing the milky fluid engages into the corneo-scleral incision first, and pressure is kept up until the nucleus of the lens rises into this bag, when one can see the pale brown nucleus with its convex upper border rising upwards. Once this happens, the pressure with the hook should not be relaxed, it is kept up till the lens jumps out of the anterior chamber.

Advantages.-This method is applicable in all types of cataractous lenses situated in the normal position, provided the age of the patient is favourable. Hypermaturity is no bar to this technique; it is more suitable than the other methods, such as the forceps and erisophake delivery, in which there is always a danger of premature rupture of a hypermature capsule before the suspensory ligament can be broken.

Complications (see Table, opposite).- The usual complications of cataract extraction are no more frequent with this technique than with any other. The dreaded danger of vitreous prolapse is slight.

Corneo-Scleral Sutures.-These are not used as a routine procedure because of the enormous amount of work handled in a single day. The corneoscleral suturing requires time, hence it is not practicable in every case. We use stitches in patients of nervous temperament, and in those suffering from high blood pressure or chronic respiratory trouble. The loops of the sutures are kept loose to avoid any interference with the delivery of the lens. 
TABLE

COMPLICATIONS ENCOUNTERED IN 1,033 CASES

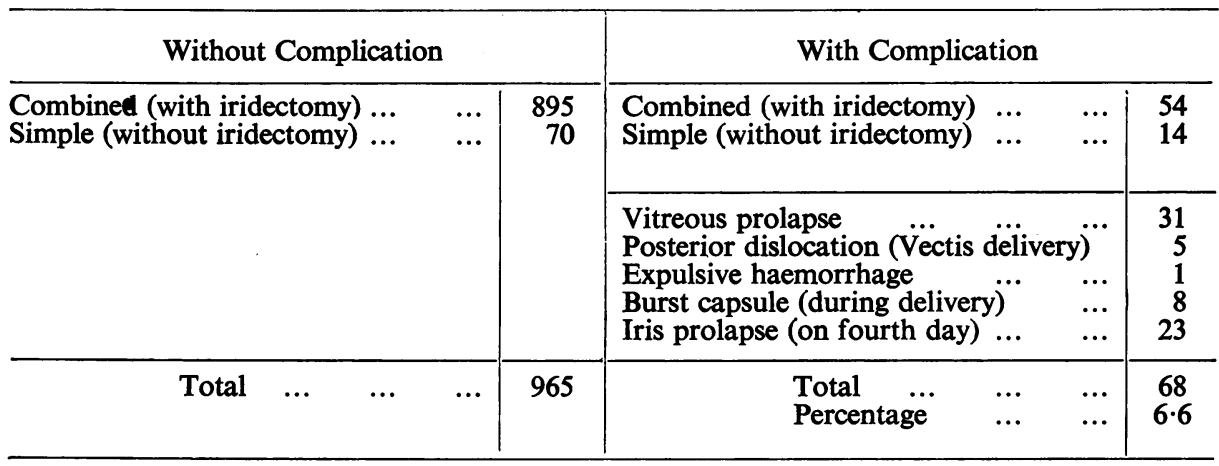

Grieshaber pattern needles $(7 \mathrm{~mm}$.) with $6 / 0$ black silk are used for this purpose.

\section{Summary}

A modification of the classical Smith Indian technique of cataract extraction is described. It is a simple procedure, involving the mechanism of lever, ledge, and tumble. All the manoeuvres are confined to the lens. It is a quick operation, with minimum complications and good results comparable with other methods of intracapsular surgery. The indications and contraindications of this procedure are reviewed.

\section{REFERENCES}

DUKe-ELDER, S. (1940). “Text-book of Ophthalmology", vol. 3, p. 3115. Kimpton, London. DuTt, K. C. (1938). Arch. Ophthal. (Chicago), 20, 1. (Quoted by Kirby, 1950.)

KIRBY, D. B. (1950). "Surgery of Cataract", pp. 1-35. Lippincott, Philadelphia.

MACNAMARA, N. C. (1868). "A Manual of the Diseases of the Eye", Churchill, London. (Cited by Smith, 1928, p. 207.)

SмITH, H. (1928). "The Treatment of Cataract", 2nd ed.; pp. 99-123. Butterworth (India), Calcutta. 